J. P. Sieb

Neurologische Klinik \& Klinik für Geriatrie und, Frührehabilitation, HELIOS HanseklinikumStralsund, Stralsund, Deutschland

\title{
CK-Serumaktivität und Epilepsie
}

\section{Erwiderung}

Zum Leserbrief von G. Krämer (2021) Postikale Hyper-CK-Ämie und antiepileptikainduzierte Myopathie bzw. Rhabdomyolyse nicht vergessen! DGNeurologie. https://doi.org/10.1007/s42451-021-00324-8

\section{Originalbeitrag}

Sieb JP (2021) Erhöhte Kreatinkinase-Serumaktivität (Hyper-CK-Ämie). DGNeurologie 4: 31-34. https://doi.org/10.1007/s42451-020-00287-2

Mein herzliches Dankeschön für das Interesse an der SOP zur Hyper-CK-Ämie (CK: Kreatinkinase) [2]. Der Leserbrief bestätigt die praktische Relevanz des zeitlichen Verlaufs der CKSerumaktivität nach einer muskulären Belastung, wie durch einen epileptischen Anfall. Physiologisch steigt die CK-Serumaktivität erst nach Stunden an, erreicht das Maximum nach 1-4 Tagen und normalisiert sich innerhalb von 3-8 Tagen. Dies muss selbstredend auch bei der Diagnostik von Anfallsereignissen berücksichtigt werden.

Zahlreiche Medikamente, wie auch Antikonvulsiva, sollen myotoxisch wirken. Hier will ich auf 2 eigene Übersichtsarbeiten verweisen $[3,4]$. Die Evidenzlage bei den einzelnen Medikamenten ist jedoch unterschiedlich. Besonders interessant ist sicher, dass der Einsatz von Valproat die klinische Manifestation einer vorbestehenden mitochondrialen Myopathie auslösen kann [1]. Auch daran muss gedacht werden.

Literatur

1. Chaudhry N, Patidar Y, Puri V (2013) Mitochondrial myopathy, encephalopathy, lactic acidosis, and stroke-like episodes unveiled by valproate. J Pediatr Neurosci 8:135-137

2. Sieb JP (2021) Erhöhte Kreatinkinase-Serumaktivität (Hyper-CK-Ämie). DGNeurologie 4:31-34

3. Sieb JP (2004) Myopathies due to drugs, toxins and nutritional deficiency In. In: Engel AG, Franzini-Armstrong C (Hrsg) Myology. McGraw-Hill, New York, S 1693-1712

4. Sieb JP, Gillessen T (2003) latrogenic and toxic myopathies. Muscle Nerve 27:142-156
Korrespondenzadresse

Prof. Dr. J. P. Sieb

Neurologische Klinik \& Klinik für Geriatrie und,

Frührehabilitation

HELIOS HanseklinikumStralsund

Große Parower Straße 47-53, 18435 Stralsund,

Deutschland

joern-peter.sieb@helios-gesundheit.de

Interessenkonflikt. J.P. Sieb gibt an, dass kein Interessenkonflikt besteht. 